\title{
Microstructural Characterization and Electrochemical Behaviour of TiC-reinforced Mo-based Composites
}

\author{
Renheng Han ${ }^{l}$, Qinyang Zeng ${ }^{1}$, Yanqin Zhu ${ }^{l}$, Ziming Bao ${ }^{l}$, Ming Tang ${ }^{1,2}$, \\ Hexin Zhang ${ }^{1,2, *}$, Chengzhi Zhao ${ }^{1,2, *}$ \\ ${ }^{1}$ College of Materials Science and Chemical Engineering, Harbin Engineering University, Harbin \\ 150001, China \\ ${ }^{2}$ Key Laboratory of Superlight Materials and Surface Technology, Ministry of Education, Harbin, \\ Engineering University, Harbin 150001, China \\ *E-mail: zhanghx@hrbeu.edu.cn
}

doi: $10.20964 / 2020.12 .79$

Received: 19 July 2020 / Accepted: 23 September 2020 / Published: 31 October 2020

\begin{abstract}
This article reports on using the spark plasma sintering (SPS) process to prepare a TiC/Mo-based alloy. The engineering application performance of the alloy was evaluated using X-ray diffraction (XRD) and transmission electron microscope (TEM) characterization methods. The electrochemical corrosion behaviour of the $\mathrm{TiC} / \mathrm{Mo}$ alloy was studied by potential polarization, and the microstructural characteristics of corrosion products were characterized and analysed using scanning electron microscopy (SEM) and energy dispersive spectrometry (EDS). The electrochemical behaviours of TiC/Mo alloys within a variety of different corrosive environments were examined, revealing the influence of corrosion resistance and the corrosion mechanism. The results showed that the TiC/Mo composite prepared by the SPS method had excellent engineering properties and met the needs of practical applications. With increasing $\mathrm{pH}$, the electrochemical corrosion rate of the TiC/Mo alloy gradually increased, as did the corrosion tendency. The surface corrosion of the TiC/Mo alloy gradually strengthened, and an oxidation-reduction reaction occurred under the action of electric current. Oxides in the form of films and agglomerated structures were formed to cover the surface of the alloy matrix. Corrosion was caused by pitting at places where alloying elements were added as well as impurity-rich areas, irregular surfaces and narrow cracks. As the degree of corrosion intensified, the corrosion pits gradually changed from black or dark brown ring-shaped spots to a vortex-like uneven corrosion morphology. The confluence of corrosion vortices further intensified the corrosion of the alloy surface. TiC/Mo alloys have good practical engineering applications and corrosion resistance within lowconcentration alkaline environments.
\end{abstract}

Keywords: TiC; Molybdenum alloy; electrochemical behaviour; polarization curves; AC impedance spectra 


\section{FULL TEXT}

(C) 2020 The Authors. Published by ESG (www.electrochemsci.org). This article is an open access article distributed under the terms and conditions of the Creative Commons Attribution license (http://creativecommons.org/licenses/by/4.0/). 